\title{
Acute Brucellosis Treated with Trimethoprim and Sulphamethoxazole
}

\author{
S. LAL,* M.B., M.R.C.P., M.R.C.P.ED. ; K. K. MODAWAL, † M.B., B.S. ; A. S. E. FOWLE, $\$ M.D., M.R.C.P. \\ B. PEACH, $\ddagger$ M.D., M.R.C.PATH. ; R. D. POPHAM, $\ddagger$ M.R.C.PATH.
}

British Medical fournal, 1970, 3, 256-257

\begin{abstract}
Cummary: Four patients with acute brucellosis are $\checkmark$ described, none of whom had any connexion with farming or milk industry, the source of infection being different in each case. The diagnosis was made by serological tests, and in three of the four cases was confirmed by positive cultures from bone marrow (one case) and liver biopsy (two cases). Treatment with the combination of trimethoprim/sulphamethoxazole was successful in three out of four cases, and in the fourth case failure may have been due to the development of trimethoprim resistance.
\end{abstract}

\section{Introduction}

Infections with organisms of the Brucella group are characterized by intracellular parasitization of reticuloendothelial cells. This phenomenon impedes the access of antibiotics and probably accounts for the chronicity which is often a feature of the infection. An antibacterial combination recently made available contains a substance-trimethoprim-with unusual penetrative powers into tissue. Combined with sulphamethoxazole it has an in-vitro activity against Brucella species. These properties prompted us to use this combination in cases of acute brucellosis. Except for a short experimental period in the first case Septrin tablets each containing $80 \mathrm{mg}$. of trimethoprim and $400 \mathrm{mg}$. of sulphamethoxazole were used. We report here our experience with the treatment of four patients, who have been followed long enough for safe conclusions to be drawn.

The patients, three men and one woman, were aged 20 to 33 years. None had special diagnostic interest, and none was connected with agriculture. They had all recently drunk unpasteurized milk, each from a different farm; they had a two to six weeks' history of malaise, anorexia, aching limbs, headache, nightmares and vague abdominal pains. They all had fevers ranging between 100 and $104^{\circ} \mathrm{F}$. ( 37.8 and $40^{\circ} \mathrm{C}$.) Two also had rigors. The liver was slightly enlarged and tender in all four. The spleen was just palpable in two. Three had enlarged and tender lymph nodes.

\section{Diagnostic Details}

The blood levels of haemoglobin, bilirubin, and aspartate and alanine aminotransferases were normal. The white cell count was low though within normal limits, but all patients had a relative lymphocytosis. Blood cultures were sterile in all four cases. Brucella abortus, however, was recovered from culture of bone marrow in the first patient and from liver tissue in the second and fourth patients. The organisms were sensitive to streptomycin, sulphonamides, and tetracycline. The minimum inhibitory concentrations (M.I.C.) for trimethoprim and sulphamethoxazole are shown in the Table. Cultures from the fourth patient were inadvertently destroyed before the sensitivities could be tested. Liver biopsies of all four patients showed classical tuberculoid granulomata.

The serological tests included were the standard agglutination test with and without the presence of 2-mercaptoethanol, antihuman globulin, and complement fixation. The tech-

* Consultant Physician, Bury General Hospital, Bury, Lancashire. † Senior House Officer in Medicine, Bury General Hospital, Bury, Lancashire.

¥ Consultant Pathologist, Bury General Hospital, Bury, Lancashire. W Wellcome Research Laboratories, Beckenham, Kent.

\begin{tabular}{|c|c|c|c|c|}
\hline \multirow{2}{*}{$\begin{array}{l}\text { Case } \\
\text { No. }\end{array}$} & \multicolumn{2}{|c|}{ Single Drugs } & \multicolumn{2}{|c|}{ Combination } \\
\hline & Sulphamethoxazole & Trimethoprim & $\begin{array}{c}\text { ISulphamethoxazole }+ \\
20 \text { parts }\end{array}$ & + Trimethoprim \\
\hline $\begin{array}{ll}1 & \ldots \\
2 & \ldots\end{array}$ & $\begin{array}{l}3 \cdot 0 \\
3 \cdot 0\end{array}$ & $\begin{array}{l}15 \cdot 0 \\
15 \cdot 0\end{array}$ & $\begin{array}{l}1.0 \\
1.0\end{array}$ & $\begin{array}{l}0.05 \\
0.05\end{array}$ \\
\hline
\end{tabular}

niques of their performance and their interpretation were as described by Kerr et al. (1968). The tests were positive in all four cases. They were carried out at intervals, and the results were used to decide whether recovery or progression to the chronic state had occurred according to the criteria of Kerr et al. (1968).

\section{Treatment and Progress}

Treatment of the first patient was, naturally, tentative and geared to changing clinical signs; hence it was complex. It is shown diagrammatically in the Chart, together with the treat-
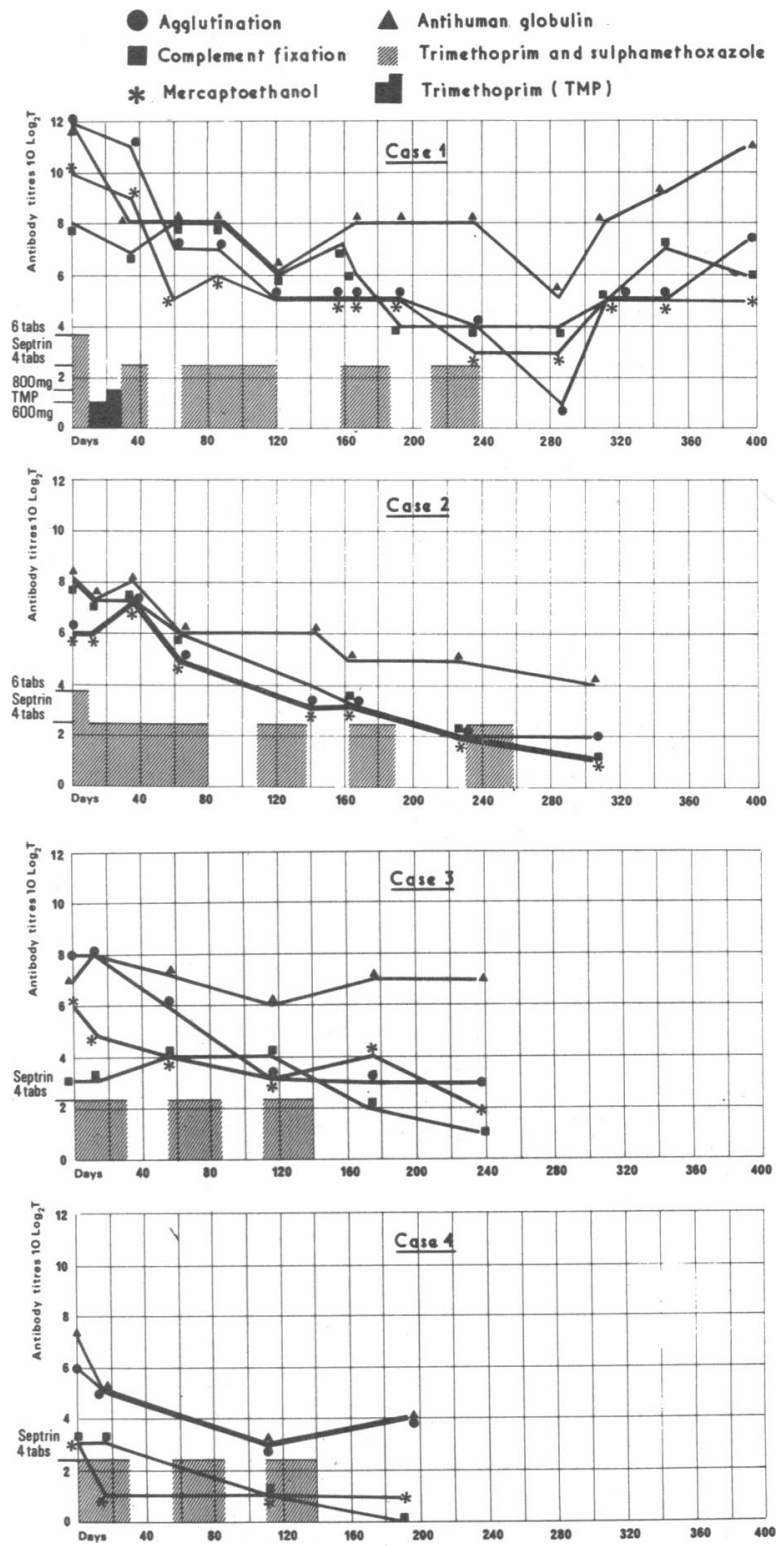
ment of the other patients and their serology. In the first sixweek period he received two tablets of Septrin thrice daily for the first 10 days and two twice daily for the last 16 days. In the interval he received trimethoprim alone. This was because on the tenth day an eruption occurred which was attributed to sulphamethoxazole, though a Herxheimer reaction was considered. He was therefore given $200 \mathrm{mg}$. of trimethoprim thrice daily. When seven days later a spike of fever recurred the dose was increased to $200 \mathrm{mg}$. four times daily. When the bacterial sensitivity results became available, however, the high M.I.C. of trimethoprim indicated that it was unwise to use this drug without its synergist. Accordingly the combination of drugs was restarted. Incidentally, it was uneventful. At the end of the projected course the serum antibody titres had begun to fall. In the eighth week he had a fever and influenza-like symptoms in circumstances which made this diagnosis quite possible. Nevertheless, he was treated with two tablets of Septrin twice daily for a further eight weeks, and then for two more periods of four weeks separated by intervals of four weeks. In all, he received 20 weeks of therapy in a period of 34 weeks.

Four months after stopping the last course the titres increased, suggesting progression to chronic brucellosis. Possibly the period of 18 days' treatment with trimethoprim alone caused bacteriological resistance to that substance.

The second patient's treatment began too early to make use of previous experience. Therefore he also received a first course of treatment perhaps longer than was necessary. He took four tablets daily for eight weeks. He then had three more courses each of four weeks. The pyrexia settled within 48 hours after starting treatment. He became symptomless within 24 hours and has remained symptom-free for over a year, and serological tests are also satisfactory.

Both the third and fourth patients were given three courses of four tablets daily for four weeks. The courses were separated by intervals of four weeks. Both have recovered, symptomatically and serologically, and the time course of their symptoms and pyrexia were like those of the second patient. In answer to direct questioning the last two patients agreed to having had some lassitude in the first treatment interval but not during the second.

\section{Discussion}

The three successful isolations of the organism out of four consecutive patients require comment. Dalrymple-Champneys (1960) recommended frequent blood cultures despite the low incidence of success which usually obtains when the species is Br. abortus and not melitensis or suis. Our own experience is similar; blood culture was unsuccessful in all four patients. The bone marrow culture, however, was successful in the first case, and so was the liver specimen culture in the second and fourth cases. These procedures are therefore recommended in future cases.

According to Robertson (1967) at least 300 herds in the county in which this hospital is situated provide for the public raw milk which is at risk of contamination with brucellae. Hence there is strong circumstantial evidence for brucellosis. In all four cases the serum titres in the tests used resembled those used as illustrations in the definitive paper on serological diagnosis by Kerr et al. (1968). The isolation of the organism in three of our cases confirms the value and accuracy which those authors ascribe to their serological criteria.

The difference between the clinical outcome of treatment and that predicted for it by in-vitro testing is particulary wide for brucella infections. This is probably due to intracellular location of the parasite as suggested by Castaneda and Carrillo-Cardenas (1953), among others. That trimethoprim penetrates cells as described by Hitchings et al. (1966) suggests that this discrepancy in prediction and actual outcome might be reduced. Though sulphonamide probably does not leave extracellular fluid to the same extent, its lower M.I.C. for brucella makes a clinical trial of the combination very attractive.

The lack of ultimate success in the first patient might have arisen from an increase in trimethoprim resistance developing through the use of the drug alone. Thus for practical purposes his subsequent treatment may have been mainly with sulphamethoxazole. In view of the modest success rate with sulphonamides alone (Spink, 1956) it would not be surprising that the patient relapsed, if trimethoprim resistance had supervened. The other three patients did well on the treatment. The standard treatment recommended at present is prolonged and repeated courses of tetracycline, supplemented with streptomycin. Treatment with Septrin is unlikely to entail more danger or discomfort than such treatment. The continuance of therapy after an eruption probably due to sulphonamide but without recurrence is therefore noteworthy. The rash appeared after 10 days' therapy and was accompanied by pyrexia. A Herxheimer reaction was considered, but sulphonamide sensitivity seemed more likely because the rash was typical. Obviously the seriousness of the original disease is an important factor in whether to start treatment again or not. The M.I.C. of sulphamethoxazole and trimethoprim individually and in combination were examined for 18 other strains of $B r$. abortus, and all were similar to those found for the organisms recovered from the patients (Bushby, 1970). This suggests that the regimen described here has a fair chance of success if used by others. As experience so far is small the necessary duration of Septrin therapy is still unknown.

Bushby and Hitchings (1968) provided data on the cidal properties of Septrin. This together with the enhanced powers of reaching the organism suggests that the duration of treatment could be shorter than those recommended for other agents. Nevertheless, the patients should be followed up for prolonged periods, with a regular check of serological tests. The treatment schedules described probably represent the maximum necessary. Nevertheless the treatment of chronic than of acute brucellosis is much more difficult. It must therefore be emphasized that the cases described here were acute. The difficulties of diagnosing and treating the chronic cases as they present in Great Britain are different, and experiences of the effect of Septrin in them should be interesting.

We are grateful to Dr. L. Robertson, consultant pathologist, Preston Roval Infirmary, for serological investigations and helpful advice; to Dr. M. N. Alefi, resident clinical pathologist, who cultured the bone marrow of the first patient; to Mr. Arnold Boulter, chief technician in the bacteriological department, for painstaking efforts in obtaining positive cultures; and to Dr. S. R. M. Bushby, of Wellcome Research Laboratories, Beckenham, Kent, for bacterial sensitivity studies.

\section{REFERENCES}

Bushby, S. R. M. (1970). Trimethoprim and sulphonamides. Laboratory studies. South African Medical fournal Supplement. In press

Bushby, S. R. M., and Hitchings, G. H. (1968). British fournal of Pharmacology and Chemotherap. $, 33,72$.

Castaneda, M. R., and Carrillo-Cardenas, C. (1953). American Gournal of the Medical Sciences, 226, 504.

Dalrymple-Champneys, W. (1960). Brucella Infection and Undulant Fever in Man. London, Oxford University Press.

Hitchings, G. H., Burchall, I. J., and Ferone, R. (1966). In Biochemical Studies of Antimicrobial Drugs, ed. B. A. Newton and P. E. Reynolds, p. 294. London, Cambridge University Press.

Kerr, W. R., et al., (1968). Fournal of Medical Microbiology, 1, 181.

Robertson, L. (1967). Fournal of Clinical Pathology, 20, 199. Spink, W. W. (1956). The Nature of Brucellosis. Minneapolis, Minnesota,
University of Minnesota Press. 